\title{
Got CPAP? Use it in the hospital!
}

\author{
Leon Rosenthal
}

Received: 3 October 2011 /Revised: 3 October 2011 / Accepted: 8 November 2011 / Published online: 25 November 2011

(C) Springer-Verlag 2011

In the present issue of the journal, Sorscher and Caruso [1] present data on the provision of continuous positive airway pressure (CPAP) during hospitalization in a cohort of 195 people. Only $26 \%$ of people with an established diagnosis of Obstructive Sleep Apnea (OSA) received CPAP therapy during hospitalization. Only obesity was found to be a predictor of receiving an admitting order for CPAP therapy. Such a low rate of treatment adherence is shocking and should prompt us to reflect on the status of treatment of OSA among hospitalized patients.

Admittedly, the report by Sorscher and Caruso [1] is based on a retrospective study of a relatively small cohort of people over a short period of time. The only other study of a similar nature was published in this journal in 2008 . The study by Spurr et al. [2] found that $5.8 \%$ of inpatients with OSA received treatment during hospitalization. As Sorscher and Caruso properly discuss in their paper, methodological limitations might have resulted in an underestimation of CPAP use in the Spurr study (as the provision of CPAP therapy was based on procedural billing codes, and thus, people using their own CPAP device from home would not have been counted as receiving therapy).

The report by Sorscher and Caruso [1] is drawn from 195 consecutive admissions of people with a preexisting diagnosis of OSA. They represented $3.2 \%$ of total admissions during the study interval. The report does not provide data on the overall characteristics of those admitted to the inpatient setting, and thus, it is not possible to comment on

L. Rosenthal $(\bowtie)$

Sleep Medicine Associates of Texas,

Dallas, TX, USA

e-mail: LRosenthal@sleepmed.com the frequency of the diagnosis for those being hospitalized during the study period. While of potential interest, it is certainly not central to the main objective of the study.

Unfortunately, the report does not include data on the severity of OSA. In the absence of this information, it is difficult to place the results of the study in the proper perspective. Categorization of the severity of OSA (and the likelihood of receiving CPAP therapy) would be of great help in evaluating the significance of the report. The binary categorization of presence or absence of OSA as an initial strategy to study the provision of CPAP in the inpatient setting is understandable as an initial strategy. However, additional analyses incorporating the severity of OSA would be most desirable. In this context, one can only speculate if obesity might have represented a proxy for the severity of OSA (or alternatively simply a clinical feature further emphasizing the desirability to treat the condition).

The lack of information about the severity of OSA paired with the seemingly inconsequential effects of not treating the condition (for most of the people in the cohort) suggests that nothing was lost by not providing therapy during the inpatient stay. This conclusion would seem legitimate given the comparable length of stay among those with and without the diagnosis of OSA. Such an outcome contrasts sharply with our clinical beliefs and multiple clinical and research reports [3, 4]. In fact, the potentially negative impact of untreated OSA in perioperative risk has prompted the American Society of Anesthesiologists to identify guidelines for preoperative assessment and screening to reduce surgical risk [5]. This paradigm prompts two considerations. One relates to additional research to further determine the negative consequences of leaving OSA untreated during nonsurgical hospitalization (clinically, this would seem unnecessary given the overwhelming evidence of negative outcomes among those with untreated OSA). 
The second consideration, which requires prompt action by clinicians, is the adoption of viable strategies to assure continued therapy of OSA during hospitalization. Failure to implement such strategies contrasts sharply with the efforts at identifying undiagnosed OSA in the preoperative stages of surgical cases. Clearly, more needs to be done to address untreated OSA in the inpatient setting.

\section{Conflict of interest None.}

\section{References}

1. Sorscher AJ, Caruso EM (2011) Frequency of provision of CPAP in the inpatient setting: an observational study. Sleep Breath (in press)
2. Spurr KF, Graven MA, Gilbert RW (2008) Prevalence of unspecified sleep apnea and the use of continuous positive airway pressure in hospitalized patients, 2004 National Hospital Discharge Survey. Sleep Breath 12(3):229-234

3. Yasuyuki K, Hajek VE, Vera Z, Raboud J, Douglas Bradley T (2003) Relationship of sleep apnea to functional capacity and length of hospitalization following stroke. Sleep 26(3):293297

4. Gupta RM, Parvizi J, Hanssen AD, Gay PC (2001) Postoperative complications in patients with obstructive sleep apnea syndrome undergoing hip or knee replacement: a case-control study. Mayo Clin Proc 76(9):897-905

5. Gross JB, Bachenberg KL, Benumof JL, Caplan RA, Connis RT, Coté CJ, Nickinovich DG, Prachand V, Ward DS, Weaver EM, Ydens L, Yu S (2006) Practice guidelines for the perioperative management of patients with obstructive sleep apnea: a report by the American Society of Anesthesiologists Task Force on Perioperative Management of patients with obstructive sleep apnea. Anesthesiology 104(5):1081-1093 